\title{
The impact of acute preoperative beta-blockade on perioperative cardiac morbidity and all-cause mortality in hypertensive South African vascular surgery patients
}

\author{
Y Moodley, MMedSci; B M Biccard, MB ChB, FCA (SA), FFARCSI, MMedSci, PhD \\ Perioperative Research Group, Department of Anaesthetics, School of Clinical Medicine, College of Health Sciences, Nelson R Mandela \\ School of Medicine, University of KwaZulu-Natal, Durban, South Africa
}

Corresponding author: Y Moodley (moodleyyo@ukzn.ac.za)

\begin{abstract}
Background. Acute $\beta$-blockade has been associated with poor perioperative outcomes in non-cardiac surgery patients, probably as a result of $\beta$-blocker-induced haemodynamic instability during the perioperative period, which has been shown to be more severe in hypertensive patients.

Objective. To determine the impact of acute preoperative $\beta$-blockade on the incidence of perioperative cardiovascular morbidity and allcause mortality in hypertensive South African (SA) patients who underwent vascular surgery at a tertiary hospital.

Methods. We conducted two separate case-control analyses to determine the impact of acute preoperative $\beta$-blockade on the incidence of major adverse cardiovascular events (MACEs, a composite outcome of a perioperative troponin-I leak or all-cause mortality) and perioperative troponin-I leak alone. Case and control groups were compared using $\chi^{2}$, Fisher's exact, McNemar's or Student's $t$-tests, where applicable. Binary logistic regression was used to determine whether acute preoperative $\beta$-blocker use was an independent predictor of perioperative MACEs/troponin-I leak in hypertensive SA vascular surgery patients.

Results. We found acute preoperative $\beta$-blockade to be an independent predictor of perioperative MACEs (odds ratio (OR) 3.496; 95\% confidence interval (CI) 1.948 - 6.273; $p<0.001$ ) and troponin-I leak (OR 5.962; 95\% CI 3.085 - 11.52; $p<0.001$ ) in hypertensive SA vascular surgery patients.

Conclusions. Our findings suggest that acute preoperative $\beta$-blockade is associated with an increased risk of perioperative cardiac morbidity and all-cause mortality in hypertensive SA vascular surgery patients.
\end{abstract}

S Afr Med J 2015;105(6):476-479. DOI:10.7196/SAMJ.8856

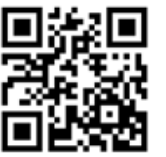

Beta-blockers are prescribed as fourth-line treatment options for hypertension in South African (SA) patients. ${ }^{[1,2]}$ Withdrawal of chronic $\beta$-blockade during the perioperative period is associated with poor outcomes in non-cardiac surgery patients. ${ }^{[3]}$ However, acute initiation of $\beta$-blockade during the perioperative period has also been shown to be associated with a higher risk of adverse outcomes following non-cardiac surgery, ${ }^{[4,5]}$ probably as a consequence of perioperative haemodynamic instability modulated by acute $\beta$-blockade. ${ }^{[6]}$ A large percentage of SA vascular patients have mean blood pressures above the therapeutic target. ${ }^{[7]}$ Acknowledging that poorly controlled hypertension is highly prevalent in the SA vascular patient population, it is possible that uncontrolled hypertension could further aggravate haemodynamic instability caused by acute $\beta$-blockade, resulting in high levels of perioperative cardiac morbidity and mortality.

\section{Objective}

To determine the impact of acute preoperative $\beta$-blockade on the incidence of perioperative cardiovascular morbidity and all-cause mortality in hypertensive SA patients who underwent vascular surgery at a tertiary hospital.

\section{Methods}

\section{Study design, setting, and patients}

This study consisted of two separate case-control analyses, and was a substudy of the South African Vascular Surgical-Cardiac Risk Index (SAVSCRI) study. ${ }^{[8]}$ Briefly, the SAVS-CRI study was a prospective cohort study conducted at Inkosi Albert Luthuli Central Hospital in Durban, SA, which sought to determine risk factors for perioperative major adverse cardiovascular events (MACEs) in vascular surgery patients. The study was approved by the University of KwaZulu-Natal Biomedical Research Ethics Committee (Protocol BF068/07, BCA117/010). A registry of consenting adult patients who underwent elective vascular surgery at the hospital between February 2008 and March 2011 was created to prospectively collect data related to demographic variables, medication use, and established risk factors for the primary outcome of this study, perioperative MACEs in vascular surgery patients receiving acute preoperative $\beta$-blockade. We did not exclude patients with respiratory, endocrine or neurological disorders from the registry, except when they were unable to provide appropriate written informed consent for inclusion in the registry. A MACE was defined as a composite of allcause mortality within 30 days of surgery or a perioperative troponin-I leak of $\geq 0.1 \mathrm{ng} / \mathrm{ml}$ that occurred within 3 days after a patient's surgery. We defined patients receiving acute preoperative $\beta$-blockade as those who had a $\beta$-blocker prescribed or administered during the same hospital admission for surgery. The definitions of established risk factors for adverse perioperative outcomes used in the SAVS-CRI study ${ }^{[8]}$ and in this study were adopted from the study of Lee et al. . $^{[9]}$ We filtered the SAVS-CRI cohort for vascular surgery patients with a history of hypertension (patients diagnosed with hypertension by a doctor, or patients taking antihypertensive medications).

\section{Matching of cases and controls}

Propensity scores for all hypertensive patients were calculated following matching according to demographic factors (age, gender), clinical 


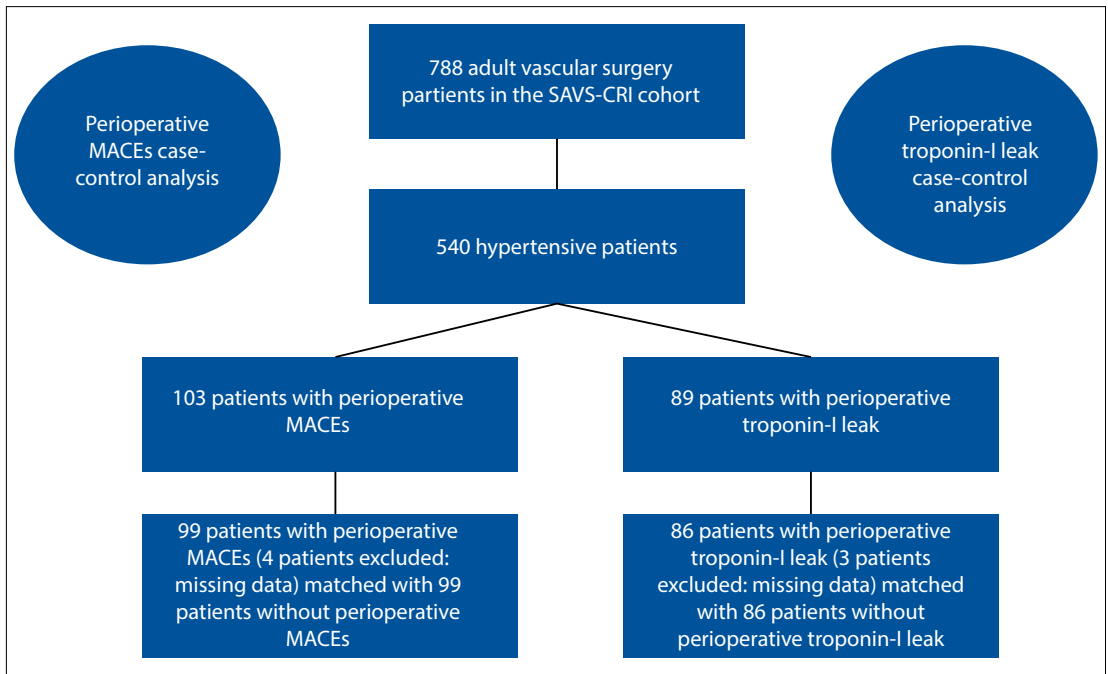

Fig. 1. Study profiles of the perioperative MACEs and perioperative troponin-I leak case-control analyses.

factors (ischaemic heart disease, diabetes, cerebrovascular disease, renal impairment and congestive heart failure), and antihypertensive medication use (angiotensin-converting enzyme inhibitors and calcium channel blockers, but not $\beta$-blockers). For the perioperative MACEs case-control analysis, cases were defined as patients who suffered perioperative MACEs, while controls were defined as patients who did not suffer perioperative MACEs. Patients were matched in a 1:1 case-to-control ratio based on similar propensity scores, resulting in a total of 99 matched pairs that were included in the analysis for the MACEs case-control analysis.

We also conducted a case-control analysis to determine the impact of acute preoperative $\beta$-blockade on the incidence of perioperative troponin-I leak alone in hypertensive SA vascular surgery patients. Patients were matched using a methodology similar to that described for the MACEs case-control analysis, resulting in a total of 86 matched pairs that were included in the case-control analysis.

\section{Statistical analysis}

Categorical data were analysed using $\chi^{2}$, Fisher's exact or McNemar's tests, where appropriate. Student's $t$-tests were used to analyse continuous data. A binary logistic regression model was used to determine whether acute preoperative $\beta$-blockade analysis for the perioperative troponin-I leak was a predictor of perioperative MACEs or perioperative troponin-I leak. Results for the categorical data analysis are presented as frequencies and percentages, while results for the binary logistic regression analyses are presented as odds ratios (ORs) with $95 \%$ confidence intervals (CIs). Any $p$-value $<0.05$ was considered to be statistically significant. All statistical analyses, including the derivation of the propensity scores for each patient, were performed using the Statistical Package for the Social Sciences (SPSS), version 21 (SPSS Inc., USA).

\section{Results}

The study profiles for both the perioperative MACEs and the perioperative troponin-I leak case-control analyses are illustrated in Fig. 1. A total of 540/788 patients from the SAVS-CRI cohort (68.5\%) were hypertensive and were screened for inclusion in the matching process. There were 103 perioperative MACEs and 89 perioperative troponin-I leaks in these 540 patients. Four patients with perioperative MACEs and 3 with perioperative troponin-I leak were excluded owing to missing comorbidity data, namely preoperative serum creatinine measurements, which were required to match cases and controls based on the criterion for renal impairment of serum creatinine $>177 \mu \mathrm{mol} / \mathrm{l}$ as defined by Lee et al. ${ }^{[9]}$ The final study population for the perioperative MACEs case-control analysis therefore consisted of 99 matched pairs of cases and controls $(n=198)$. A total of $31 / 99$ patients (31.4\%) had died within 30 days of surgery,

Table 1. Clinical characteristics of cases and controls in the perioperative MACEs case-control analysis

\begin{tabular}{|c|c|c|c|c|}
\hline Clinical characteristic & All patients $(N=198)$ & $\begin{array}{l}\text { Patients with perioperative } \\
\text { MACEs (cases, } N=99 \text { ) }\end{array}$ & $\begin{array}{l}\text { Patients without perioperative } \\
\text { MACEs (controls, } N=99 \text { ) }\end{array}$ & $p$-value ${ }^{*}$ \\
\hline Male gender, $n(\%)$ & $103(52.0)$ & $53(53.5)$ & $50(50.5)$ & 0.670 \\
\hline Age (years), mean (SD) & $64.4(11.4)$ & $64.6(12.3)$ & $64.2(10.3)$ & 0.793 \\
\hline Ischaemic heart disease, $n(\%)$ & $119(60.1)$ & $57(57.6)$ & $62(62.6)$ & 0.468 \\
\hline Diabetes, $n(\%)$ & $134(67.7)$ & $64(64.6)$ & $70(70.7)$ & 0.362 \\
\hline Congestive heart failure, $n(\%)$ & $21(10.6)$ & $10(10.1)$ & $11(11.1)$ & 0.817 \\
\hline Renal impairment, $n(\%)$ & $8(4.0)$ & $4(4.0)$ & $4(4.0)$ & 1.000 \\
\hline History of cerebrovascular accident, $n(\%)$ & $42(21.2)$ & $21(21.2)$ & $21(21.2)$ & 1.000 \\
\hline High-risk surgical procedures, $n(\%)$ & $28(14.1)$ & $15(15.2)$ & $13(13.1)$ & 0.683 \\
\hline Acute preoperative aspirin use, $n(\%)$ & $190(96.0)$ & $94(94.9)$ & $96(97.0)$ & 0.721 \\
\hline $\begin{array}{l}\text { Acute preoperative calcium channel } \\
\text { blocker use, } n(\%)\end{array}$ & $69(34.8)$ & $37(37.4)$ & $32(32.3)$ & 0.456 \\
\hline $\begin{array}{l}\text { Acute preoperative angiotensin- } \\
\text { converting enzyme inhibitor use, } n(\%)\end{array}$ & $139(70.2)$ & $70(70.7)$ & $69(69.7)$ & 0.877 \\
\hline Acute preoperative $\beta$-blocker use, $n(\%)$ & $100(50.5)$ & $65(65.7)$ & $35(35.4)$ & $<0.001$ \\
\hline
\end{tabular}


with perioperative troponin leaks noted in 15 of these cases $(48.4 \%)$. The final study population for the perioperative troponin-I leak case-control analysis consisted of 86 matched pairs of cases and controls $(n=172)$.

The clinical characteristics of the case and control groups in the perioperative MACEs case-control analysis are shown in Table 1. With the exception of acute preoperative $\beta$-blocker use $(p<0.001)$, no statistically significant results were noted on $\chi^{2}$ testing between any other clinical characteristics in the case and control groups, indicating appropriate matching of the individual cases and corresponding controls. The results of McNemar's test also showed that the association between acute preoperative $\beta$-blocker use and perioperative MACEs was statistically significant in this study $(p<0.001)$. When entered into a logistic regression equation, acute preoperative $\beta$-blocker use was associated with an almost 3.5 -fold increased risk of developing perioperative MACEs following vascular surgery in hypertensive SA patients (OR 3.496; 95\% CI 1.948 - 6.273; $p<0.001)$.

Table 2 shows the clinical characteristics of cases and controls in the perioperative troponin-I leak case-control analysis. As with the perioperative MACEs casecontrol analysis, cases and controls in the perioperative troponin-I leak case-control analysis appeared to be efficiently matched with regard to clinical comorbidities, procedural risk, and calcium channel blocker and angiotensin-converting enzyme inhibitor use. A statistically significant association between preoperative acute $\beta$-blockade and perioperative troponin-I leak was observed following $\chi^{2}$ testing $(p<0.001)$. When case-control pairs were analysed via McNemar's test, an association between acute preoperative $\beta$-blockade and perioperative troponin-I leak was observed $(p<0.001)$. When entered into a logistic regression equation, acute preoperative $\beta$-blocker use was associated with an increased risk of developing perioperative troponin-I leak following vascular surgery in hypertensive SA patients (OR 5.962; 95\% CI 3.085 - 11.52; $p<0.001)$. A description of acute preoperative $\beta$-blocker use for both case-control analyses is presented in Table 3 .

Table 2. Clinical characteristics of cases and controls in the perioperative troponin-I leak case-control analysis

\begin{tabular}{|c|c|c|c|c|}
\hline Clinical characteristic & All patients $(N=172)$ & $\begin{array}{l}\text { Patients with perioperative } \\
\text { troponin-I leak (cases, } N=86 \text { ) }\end{array}$ & $\begin{array}{l}\text { Patients without perioperative } \\
\text { troponin-I leak (controls, } N=86 \text { ) }\end{array}$ & $p$-value ${ }^{\star}$ \\
\hline Male gender, $n(\%)$ & $87(50.6)$ & $45(52.3)$ & $42(48.8)$ & 0.647 \\
\hline Age (years), mean (SD) & $63.8(11.1)$ & $64.2(12.2)$ & $63.4(9.9)$ & 0.657 \\
\hline Ischaemic heart disease, $n(\%)$ & $109(63.4)$ & $53(61.6)$ & $56(65.1)$ & 0.635 \\
\hline Diabetes, $n(\%)$ & $121(70.3)$ & $58(67.4)$ & $63(73.3)$ & 0.404 \\
\hline Congestive heart failure, $n(\%)$ & $18(10.5)$ & $9(10.5)$ & $9(10.5)$ & 1.000 \\
\hline Renal impairment, $n(\%)$ & $6(3.5)$ & $3(3.5)$ & $3(3.5)$ & 1.000 \\
\hline $\begin{array}{l}\text { History of cerebrovascular accident, } \\
n(\%)\end{array}$ & $39(22.7)$ & $20(23.3)$ & $19(22.1)$ & 0.856 \\
\hline High-risk surgical procedures, $n(\%)$ & $27(15.7)$ & $14(16.3)$ & $13(15.1)$ & 0.834 \\
\hline Acute preoperative aspirin use, $n(\%)$ & $165(95.9)$ & $81(94.2)$ & $84(97.7)$ & 0.443 \\
\hline $\begin{array}{l}\text { Acute preoperative calcium channel } \\
\text { blocker use, } n(\%)\end{array}$ & $58(33.7)$ & $32(37.2)$ & $26(30.2)$ & 0.333 \\
\hline $\begin{array}{l}\text { Acute preoperative angiotensin- } \\
\text { converting enzyme inhibitor use, } n(\%)\end{array}$ & $114(66.3)$ & $59(68.6)$ & $55(64.0)$ & 0.519 \\
\hline Acute preoperative $\beta$-blocker use, $n(\%)$ & $88(51.2)$ & $62(72.1)$ & $26(30.2)$ & $<0.001$ \\
\hline
\end{tabular}

Table 3. Description of acute preoperative $\beta$-blocker use in both case-control analyses

\begin{tabular}{|c|c|c|c|c|c|c|}
\hline \multirow[b]{2}{*}{ Beta-blockers used } & \multicolumn{3}{|c|}{ Perioperative MACEs case-control analysis } & \multicolumn{3}{|c|}{ Perioperative troponin-I leak case-control analysis } \\
\hline & All patients & $\begin{array}{l}\text { Patients with } \\
\text { perioperative MACEs }\end{array}$ & $\begin{array}{l}\text { Patients without } \\
\text { perioperative MACEs }\end{array}$ & All patients & $\begin{array}{l}\text { Patients with } \\
\text { perioperative } \\
\text { troponin-I leak }\end{array}$ & $\begin{array}{l}\text { Patients without } \\
\text { perioperative } \\
\text { troponin-I leak }\end{array}$ \\
\hline Atenolol only, $n(\%)$ & $80(80.0)$ & $53(81.5)$ & $27(77.1)$ & $71(80.7)$ & $49(79.0)$ & $22(84.6)$ \\
\hline $\begin{array}{l}\text { Atenolol + } \\
\text { carvedilol, } n(\%)\end{array}$ & $5(5.0)$ & $3(4.6)$ & $2(5.7)$ & $5(5.7)$ & $3(4.8)$ & $2(7.7)$ \\
\hline $\begin{array}{l}\text { Atenolol + labetalol, } \\
n(\%)\end{array}$ & $2(2.0)$ & $1(1.5)$ & $1(2.9)$ & $1(1.1)$ & $1(1.6)$ & $0(0.0)$ \\
\hline $\begin{array}{l}\text { Carvedilol only, } \\
n(\%)\end{array}$ & $12(12.0)$ & $7(10.9)$ & $5(14.3)$ & $10(11.4)$ & $8(13.0)$ & $2(7.7)$ \\
\hline Labetalol only, $n(\%)$ & $1(1.0)$ & $1(1.5)$ & $0(0.0)$ & $1(1.1)$ & $1(1.6)$ & $0(0.0)$ \\
\hline Total, $n(\%)$ & $100(100.0)$ & $65(100.0)$ & $35(100.0)$ & $88(100.0)$ & $62(100.0)$ & $26(100.0)$ \\
\hline
\end{tabular}




\section{Discussion}

We found acute preoperative $\beta$-blockade to be an independent predictor of perioperative cardiac morbidity (as evidenced by perioperative troponin-I measurements) and all-cause mortality in hypertensive SA vascular surgery patients.

We suspect that these results are related to perioperative haemodynamic instability. Hypotension is a common perioperative complication in vascular surgery patients with a history of poorly controlled hypertension, with a study by Charlson et al. ${ }^{[10]}$ reporting a higher incidence of perioperative hypotension in patients who had preoperative mean arterial blood pressures $\geq 110 \mathrm{mmHg}$ than in those with preoperative mean arterial blood pressures of $<100 \mathrm{mmHg}$. In another study by Charlson et al., ${ }^{[11]}$ a decrease in mean arterial blood pressure of $\geq 20 \mathrm{mmHg}$ for $\geq 5$ minutes was associated with an increased incidence of perioperative cardiac complications in non-cardiac surgery patients. Perioperative hypotension is therefore an important determinant of perioperative cardiac outcomes in non-cardiac surgery patients, in particular those with poorly controlled hypertension.

Acute preoperative $\beta$-blockade may further aggravate the haemodynamic instability associated with a history of poorly controlled hypertension. The POISE-I study was a randomised controlled trial comparing the effect of acutely administered, extended-release $\beta$-blocker (metoprolol succinate) with that of placebo on the 30-day risk of major cardiovascular events in patients with, or at risk of, atherosclerotic disease who were undergoing non-cardiac surgery. ${ }^{[12]}$ In this study, patients randomised to acute preoperative $\beta$-blockade had significantly more hypotension. Furthermore, hypotension was associated with perioperative mortality (hazard ratio (HR) 1.33; 95\% CI 1.03 - 1.74; $p=0.0317$ ). An analysis of the causation of perioperative deaths in the POISE-I study suggested that hypotension was partly associated with some of the mortality. ${ }^{[12]}$

Although the POISE-I study ${ }^{[12]}$ did not find perioperative hypotension to be associated with perioperative myocardial infarction in non-cardiac surgery patients, the POISE-II study, ${ }^{[13]}$ which was a randomised controlled study of clonidine v. placebo, found clinically important hypotension to be independently associated with perioperative myocardial infarction (HR 1.37; 95\% CI 1.16 - 1.62; $p<0.001)^{[13]}$

It is therefore likely that our study population of hypertensive vascular surgery patients with previously documented poorly controlled hypertension may have been predisposed towards perioperative hypotension, which could have been further exacerbated by acute preoperative $\beta$-blockade, resulting in the observed increased incidence of perioperative cardiac morbidity and all-cause mortality. These findings have important implications for primary and secondary prevention of myocardial ischaemia in the perioperative period in hypertensive vascular surgery patients.

\section{Study limitations}

Our study had several limitations. We only collected data related to perioperative cardiac outcomes and all-cause mortality during this study, and we were unable to evaluate the impact of acute preoperative $\beta$-blockade on other perioperative complications that might have occurred, such as stroke and clinically important hypotension. Based on results from POISE-I, ${ }^{[12]}$ it is likely that acute preoperative $\beta$-blockade may also further increase perioperative stroke risk in SA hypertensive vascular surgical patients. We did not collect data related to chronic obstructive airway disease and were therefore unable to control for this factor in either of the case-control analyses. We also did not collect data related to neurological disorders other than cerebrovascular disease. While we did not collect data related to diabetes treatment, it is likely that diabetic patients were placed on an insulin sliding scale preoperatively, in line with current local practice. Although we did not collect information related to the actual dosage of the drugs administered to vascular patients in our institution, atenolol is commonly prescribed at a dose of $50 \mathrm{mg}$ orally per day, labetalol is titrated to effect with heart rate control when patients cannot take medications orally, and carvedilol is prescribed for heart failure and is up-titrated to the desired effect over a period of time.

In addition, we were unable to access information related to patients' cause of death once they had been discharged from hospital. Another limitation is that our patient population was drawn from a single tertiary hospital and represented a population with substantial medical comorbidity at high-risk for perioperative cardiac complications or death, requiring specialist vascular surgery. It is therefore possible that the epidemiology of acute preoperative $\beta$-blocker administration and patient comorbidities in our study population may be different from that in patients attending lower-level facilities, or even facilities in different geographical locales within SA.

\section{Conclusions}

Acute preoperative $\beta$-blocker administration was associated with an increased risk of perioperative cardiac morbidity and all-cause mortality in hypertensive SA vascular surgery patients. A cautious approach should be taken when considering initiating acute preoperative $\beta$-blockade in hypertensive surgical patients.

Acknowledgements. This study was funded by a South African Medical Research Council self-initiated research grant awarded to BMB. This work forms a component of the doctoral studies of YM, who is the recipient of a doctoral scholarship awarded by the South African National Research Foundation. YM was also supported by the Columbia University Southern African Fogarty AIDS International Training and Research Program (AITRP) Implementation Science Scholarship Program funded by PEPFAR through the Fogarty International Center, National Institutes of Health (grant \# D43TW000231).

\section{References}

1. Parker A, Nagar B, Thomas G, Badri M, Ntusi NB. Health practitioners' state of knowledge and challenges to effective management of hypertension at primary level. Cardiovasc J Afr 2011;22(4):186190. [http://dx.doi.org/10.5830/CVJA-2010-066]

2. Seedat YK, Rayner BL. South African hypertension guideline 2011. S Afr Med J 2012;102(1):57-83.

3. Shammash JB, Trost JC, Gold JM, Berlin JA, Golden MA, Kimmel SE. Perioperative beta-blocker withdrawal and mortality in vascular surgical patients. Am Heart J 2001;141(1):148-153. [http:/ dx.doi.org/10.1067/mhj.2001.111547]

4. Wijeysundera DN, Beattie WS, Wijeysundera HC, Yun L, Austin PC, Ko DT. Duration of preoperative beta-blockade and outcomes after major elective noncardiac surgery. Can J Cardiol 2014;30(2):217 223. [http://dx.doi.org/10.1016/j.cjca.2013.10.011]

5. Bouri S, Shun-Shin MJ, Cole GD, Mayet J, Francis DP. Meta-analysis of secure randomised controlled Bouri S, Shun-Shin MJ, Cole GD, Mayet J, Francis DP. Meta-analysis of secure randomised controlled
trials of beta-blockade to prevent perioperative death in non-cardiac surgery. Heart 2014;100(6):456trials of beta-blockade to prevent perioperative death

6. Devereaux PJ, Beattie WS, Choi PT, et al. How strong is the evidence for the use of perioperative beta blockers in non-cardiac surgery? Systematic review and meta-analysis of randomised controlled trials. BMJ 2005;331(7512):313-321. [http://dx.doi.org/10.1136/bmj.38503.623646.8F]

7. Brand M, Woodiwiss AJ, Michel F, et al. Chronic diseases are not being managed effectively in eithe high-risk or low-risk populations in South Africa. S Afr Med J 2013;103(12):938-941. [http://dx.doi org/10.7196/samj.6018

8. Moodley Y, Naidoo P, Biccard BM. The South African Vascular Surgical Cardiac Risk Index (SAVS-CRI): A prospective observational study. S Afr Med J 2013;103(10):746-750. [http://dx.doi. org/10.7196/samj.6967]

9. Lee TH, Marcantonio ER, Mangione CM, et al. Derivation and prospective validation of a simple index for prediction of cardiac risk of major noncardiac surgery. Circulation 1999;100(10):1043-1049. index for prediction of cardiac risk of major nonc
[ $\mathrm{http}: / / \mathrm{dx}$.doi.org/10.1161/01.CIR.100.10.1043]

10. Charlson ME, MacKenzie CR, Gold JP, Ales KL, Topkins M, Shires GT. Preoperative characteristics predicting intraoperative hypotension and hypertension among hypertensives and diabetics undergoing noncardiac surgery. Ann Surg 1990;212(1):66-81.

11. Charlson ME, MacKenzie CR, Gold JP, et al. The preoperative and intraoperative hemodynamic predictors of postoperative myocardial infarction or ischemia in patients undergoing noncardiac surgery. Ann Surg 1989;210(5):637-648.

12. Devereaux PJ, Yang H, Yusuf S, et al. Effects of extended-release metoprolol succinate in patients undergoing non-cardiac surgery (POISE trial): A randomised controlled trial. Lancet 2008;371(9627):1839-1847. [http://dx.doi.org/10.1016/S0140-6736(08)60601-7]

13. Devereaux PJ, Sessler DI, Leslie K, et al. Clonidine in patients undergoing noncardiac surgery. N Engl J Med 2014;370(16):1504-1513. [http://dx.doi.org/10.1056/NEJMoal401106]

Accepted 21 April 2015 